\title{
Mesenchymal stem cells in synovial fluid increase in knees with degenerative meniscus injury after arthroscopic procedures through the endogenous effects of CGRP and HGF
}

\section{Naoto Watanabe}

Tokyo Medical and Dental University

Kentaro Endo

Tokyo Medical and Dental University

Keiichiro Komori

Tokyo Medical and Dental University

Nobutake Ozeki

Tokyo Medical and Dental University

Mitsuru Mizuno

Tokyo Medical and Dental University

Hisako Katano

Tokyo Medical and Dental University

Yuji Kohno

Tokyo Medical and Dental University

Kunikazu Tsuji

Tokyo Medical and Dental University

\section{Hideyuki Koga}

Tokyo Medical and Dental University

Ichiro Sekiya ( $\nabla$ sekiya.arm@tmd.ac.jp )

Tokyo Medical and Dental University https://orcid.org/0000-0002-6331-722X

\section{Research}

Keywords: mesenchymal stem cell, synovial fluid, degenerative meniscus injury, migration

Posted Date: July 17 th, 2020

DOl: https://doi.org/10.21203/rs.3.rs-42459/v1

License: (c) (i) This work is licensed under a Creative Commons Attribution 4.0 International License. Read Full License 
Page $2 / 20$ 


\section{Abstract}

Background: Mesenchymal stem cells (MSCs) in synovial fluid increase after traumatic meniscus injuries. However, MSC kinetics in synovial fluid may differ for knees with degenerative meniscus injuries. Furthermore, the combination of surgical repair and synovial MSC transplantation has been found to improve clinical symptoms in patients with degenerative meniscus injury, and in this treatment, only the operation procedure without MSC transplantation might increase MSCs in synovial fluid; if so, soluble factors in synovial fluid will be involved. The purpose is this study was to examine whether MSCs exist in synovial fluid of knees with degenerative meniscus injury, to investigate whether MSCs in synovial fluid increase after harvest of synovium and meniscus repair, and to explore what soluble factors in synovial fluids affect the number of MSCs in synovial fluid.

Methods: Subjects were 7 patients with degenerative meniscus injury who underwent meniscal repair and synovial MSC transplantation. Synovial fluid (Pre) was aspirated from knees before harvest of synovium and meniscus repair. After 2 weeks, synovial fluid (Post) was aspirated again before transplantation of synovial MSCs. A half volume of the synovial fluid was plated and cultured for 2 weeks to count the colony formation. The other half was used for antibody array analysis, and the correlation coefficients between the signal intensity and colony number were measured in 503 factors. Factors with high correlation coefficients were verified by migration assay.

Results: While cell colonies derived from synovial fluid (Pre) were hardly observed, greater numbers of colonies from synovial fluid (Post) were demonstrated. Of the 503 factors, calcitonin gene-related peptide (CGRP) and hepatocyte growth factor (HGF) had high correlation coefficients between colony number and expression level. Both CGRP and HGF promoted migration of synovial fluid MSCs.

Conclusions: MSCs in synovial fluid were hardly seen in knees with degenerated meniscus injury. They significantly increased 2 weeks after harvest of synovium and meniscus repair. Both CGRP and HGF in synovial fluid can possibly induce MSCs from synovium into synovial fluid.

\section{Background}

Mesenchymal stem cells (MSCs) are a promising cell source for regenerative medicine for a wide range of applications (1). While MSCs in synovial fluid are hardly detected in normal knees, they increase after injuries of anterior cruciate ligament (2) and cartilage (3), and their gene profiles are similar to those of the synovium. A previous study reported that MSCs in synovial fluid also increase after traumatic meniscus injury (4). In animal studies, exogenous synovial MSCs promoted meniscus healing (5-9). These studies suggest that MSCs in synovial fluid increase after traumatic meniscus injury and contribute to spontaneous meniscus healing. Degenerative tears of the meniscus occur frequently in middle-aged or older persons and are usually located at the posterior horn of the medial meniscus (10). They are generally slowly developing tears without traumatic onset and typically involve a horizontal cleavage of the meniscus. As pathological conditions differ between traumatic and degenerative 
meniscus tears, the kinetics of MSCs in synovial fluid may also be different between them. The first purpose of our study is to examine whether MSCs exist in the synovial fluid of knees with degenerative meniscus injury.

Symptomatic degenerative tears are a common clinical problem, and when surgery is required, the usual choice is arthroscopic partial meniscectomy rather than meniscal repair due to the poor healing potential of the meniscus (11). However, meniscectomy increases the risk of osteoarthritis (OA) (12-14). A previous study found that the combination of surgical repair and synovial MSC transplantation improved the clinical symptoms in patients with a complex degenerative tear of the medial meniscus (15). However, the previous report suggested that agitation of the synovial surface increased synovial fluid MSCs (16). Therefore, only the operation procedure including harvest of synovium and meniscus repair might increase MSCs in synovial fluid. If so, quantifying MSCs in synovial fluid after those procedures would provide useful information for cell therapy. Thus, the second purpose of this study is to investigate whether MSCs in synovial fluid increase after harvest of synovium and meniscus repair.

Previous reports have indicated some factors in synovial fluids that are involved in traumatic and degenerative meniscus tears $(17,18)$. In addition, MSCs other than those derived from the synovium exhibit chemotaxis to various humoral factors (19-21). These suggest that recruitment of MSCs from the synovium into synovial fluid involves humoral factors in synovial fluid. However, these humoral factors were hardly investigated (17). If such humoral factors are revealed, they will lead to the development of cell-free regenerative medicine for meniscus. The third purpose of this study is to explore what humoral factors in synovial fluid affect the number of synovial fluid MSCs.

\section{Methods}

\section{Collection of Synovial Fluids}

This study was approved by the Medical Research Ethics Committee of Tokyo Medical and Dental University, and informed consent was obtained from all study subjects.

The subjects were 7 patients who had complex degenerative tears of the medial meniscus and underwent meniscal repair and synovial MSC transplantation. Their ages ranged from 29 to 60 years old. The meniscus symptoms, which included feeling instable in addition to pain, developed gradually in all patients, and the duration of the meniscus symptoms ranged from 4 months to 4 years. OA patients who were diagnosed as Kellgren-Lawrence grades 2-4 were not included. According to arthroscopic observation, all patients had complex degenerative meniscal tears, including a horizontal tear and flap tear at the avascular area of the middle and posterior segment of the medial meniscus.

Synovial fluid (Pre) was aspirated from knees with degenerative meniscus injury before harvest of approximately $0.5 \mathrm{~g}$ synovium (consisting of approximately 20 pieces by pituitary rongeur) and meniscus repair (Fig. 1). After 2 weeks, synovial fluid (Post) was aspirated again before transplantation of synovial MSCs. 


\section{Cultures of Synovial Fluid MSCs}

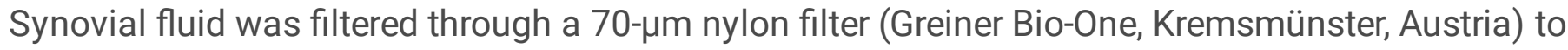
remove debris, and a half volume of the synovial fluid was plated in 6 culture dishes of $60 \mathrm{~cm}^{2}$ (Thermo Fisher Scientific, Waltham, MA, USA) in complete culture medium: a-modified essential medium (a-MEM; Thermo Fisher Scientific, Waltham, MA, USA) containing 10\% fetal bovine serum (FBS) (Thermo Fisher Scientific), $100-\mathrm{U} / \mathrm{mL}$ penicillin, $100-\mu \mathrm{g} / \mathrm{mL}$ streptomycin, and $250-\mathrm{ng} / \mathrm{mL}$ amphotericin $\mathrm{B}$ (Thermo Fisher Scientific). The dishes were incubated at $37^{\circ} \mathrm{C}$ with $5 \%$ humidified $\mathrm{CO}_{2}$. After 24 hours, the nonadherent cells were washed out with phosphate-buffered saline (PBS). Fourteen days after initial plating, 3 dishes were stained with $0.5 \%$ crystal violet (Wako, Osaka, Japan) in $4 \%$ paraformaldehyde for 10 minutes for colony formation analysis. Colonies less than $2 \mathrm{~mm}$ in diameter and faintly stained colonies were ignored. The other 3 dishes were harvested with $0.25 \%$ trypsin and 1-mM ethylenediaminetetraacetic acid (EDTA) (Invitrogen, CA, USA) (Passage 0), and the number of isolated cells were counted. Then, the cells were replated at $500 \mathrm{cells} / \mathrm{cm}^{2}$ in a $145-\mathrm{cm}^{2}$ culture dish (Thermo Fisher Scientific) and cultured for 14 days for surface epitopes and differentiation analyses.

\section{Surface Epitopes}

For synovial fluid MSCs from 7 donors, surface markers were examined using a FACSVerse instrument (Becton, Dickinson and Company; BD, NJ, USA). The cells were suspended in FACS buffer ( $2 \% \mathrm{FBS}, 5 \mathrm{mM}$ EDTA in PBS) using Hank's balanced salt solution (HBSS) at a density of $5 \times 105$ cells $/ \mathrm{mL}$ and stained for 30 minutes with the antibodies CD44 (APC-A), CD90 (PerCP-Cy5.5-A), CD73 (V450-A), CD105 (PE-A), CD31 (PE-Cy7-A), and CD45 (FITC-A) (all from BD) and Ghost Dye Violet 510 for dead cells (Tonbo Biosciences, CA, USA). BD FACSuite software (BD) was used for the analysis.

\section{Differentiation Assay}

Differentiation assay was conducted as previously described.(22) For chondrogenesis, $2.5 \times 10^{5}$ cells were transferred to a $15-\mathrm{mL}$ tube (BD Falcon) and cultured in chondrogenic induction medium containing 10-ng/mL transforming growth factor- $\beta 3$ (Miltenyi Biotec Japan, Tokyo, Japan) and 500-ng/mL bone morphogenetic protein 2 (BMP-2, Infuse; Medtronic, Minneapolis, MN); this medium was changed every 34 days. After 21 days, the cell pellets were embedded, sectioned, and stained with safranin-o and fast green (Wako, Tokyo, Japan).

Calcification was studied by plating 100 cells in a $60-\mathrm{cm}^{2}$ dish and culturing for 14 days in a-MEM with $10 \%$ FBS. Adherent cells were subsequently cultured in an osteogenic induction medium containing 50$\mu \mathrm{g} / \mathrm{mL}$ ascorbic acid 2-phosphate (Wako), 10-nM dexamethasone (Wako), and 10-mM $\beta$-glycerophosphate (Sigma-Aldrich, St Louis, MO, USA); this medium was changed every 3-4 days. After 14 days, calcification was assessed by alizarin red (Sigma-Aldrich) staining.

Adipogenesis was evaluated by plating 100 cells in a $60-\mathrm{cm}^{2}$ dish and culturing for 14 days to allow colony formation. Adherent cells were cultured in an adipogenic induction medium supplemented with 
100-nM dexamethasone, 0.5-mM isobutylmethylxanthine (Sigma-Aldrich), and 50-mM indomethacin (Sigma-Aldrich) for an additional 14 days; this medium was changed every 3-4 days. Adipocyte colonies were stained with oil red-o (Sigma-Aldrich).

\section{Antibody Array Analyses}

After filtration, the half volume of synovial fluid (Post) was cryopreserved at $-80^{\circ} \mathrm{C}$ and used to assess the expression levels of 507 proteins using RayBiotech's L-Series human antibody array L-507 membrane kit (RayBiotech, Peachtree Corners, GA, USA) according to the manufacturer's instructions. Briefly, all synovial fluid samples were diluted 100 times, followed by quantification and biotinylation. Overnight incubation of the membrane array with biological samples at $4^{\circ} \mathrm{C}$ resulted in the binding of proteins to corresponding antibodies. Signals were visualized using horseradish peroxidase (HRP)conjugated streptavidin and were imaged by Chemi Doc XRS (Bio-Rad Laboratories, Hercules, CA, USA). The final spot intensities were measured as the original intensities, subtracting the background. The data were normalized to Angiopoietin-like 1 for the positive controls, as this did not change in amount among patients. The correlation coefficients between the signal intensity and the colony number per dish were measured.

\section{Transwell Migration of Synovial Fluid MSCs}

After being cultured for 5-7 days with a-MEM containing $10 \%$ FBS, the medium was changed to a-MEM containing $0.5 \%$ FBS and incubated for 24 hours for serum starvation. A total of $5 \times 10^{4}$ synovial fluid MSCs were seeded to the upper chambers with 8- $\mu \mathrm{m}$ Transwell microporous membranes (Cell Culture Insert; Falcon, Franklin Lakes, New Jersey, USA) in 24-well plates (Falcon, Franklin Lakes, New Jersey, USA) (Fig. 2). The cells in the upper chambers were allowed to migrate across the membrane at $37^{\circ} \mathrm{C}$ and $5 \% \mathrm{CO}_{2}$. Calcitonin gene-related peptide (CGRP) (Sigma-Aldrich, St Louis, MO, USA) at a concentration of $10^{-8} \mathrm{M}, 10^{-10} \mathrm{M}$, and $10^{-12} \mathrm{M}$ or hepatocyte growth factor (HGF) (Peprotech, Rocky Hill, NJ, USA) at a concentration of $10 \mathrm{ng} / \mathrm{mL}, 1.0 \mathrm{ng} / \mathrm{mL}$, and $0.1 \mathrm{ng} / \mathrm{mL}$ was added in the lower chamber containing aMEM with $0.5 \%$ FBS. After 6 hours, the upper chambers were stained with $0.5 \%$ crystal violet in $4 \%$ paraformaldehyde for 10 minutes. MSCs that did not migrate across the pores were wiped out with a cotton swab, and the number of MSCs on the lower side of the membrane was counted by light microscopy (BZ-X700, Keyence Co., Ltd, Osaka, Japan).

\section{Statistical Analysis}

All data were statistically evaluated using GraphPad Prism 6 (GraphPad Software, La Jolla, CA, USA). All data were expressed in dot plots. Each statistical analysis method is described in the figure legends. $P$ values less than 0.05 were considered statistically significant. 


\section{Results}

\section{Colony Formation Before and After Arthroscopic Surgery}

While cell colonies derived from synovial fluid (Pre) were hardly observed, a much greater number of colonies derived from synovial fluid (Post) were demonstrated (Fig. 3A). Synovial fluid volume increased significantly 2 weeks after harvest of synovium and meniscus repair (Fig. 3B). The mean colony number per dish significantly increased from $1.9 \pm 4.5$ to $36.0 \pm 17.4$ (Fig. 3C). The mean cell number per dish increased from $15 \pm 40 \times 10^{3}$ to $370 \pm 230 \times 10^{3}$ (Fig. 3D).

\section{Properties of Colony-Forming Cells in Synovial Fluid}

Colony-forming cells in synovial fluid were mostly spindle shaped (Fig. 4A). They expressed CD44, CD73, CD 90, and CD105 at high rates and did not express CD31 and CD45 (Fig. 4B). Colony-forming cells differentiated into cartilage positively stained with safranin-o, calcified positively stained with alizarin red, and differentiated into adipocytes stained with oil red-o (Fig. 4C).

\section{Relationship Between Protein Level and Colony Number in Synovial Fluid MSCs}

Antibody array analyses showed that the signal intensity for 503 factors in synovial fluid (Post) varied in 7 donors (Fig. 5A). Of the 503 factors, 8 had a correlation coefficient between colony number and expression level of higher than 0.70 (Fig. 5B). Among these factors, those with a $p$ value less than 0.05 and commercially available were CGRP and HGF.

\section{Chemotactic Effect of CGRP and HGF on Synovial Fluid MSC Migration}

Both CGRP and HGF promoted migration of synovial fluid MSCs (Fig. 6A). Quantification analyses showed that $10^{-8} \mathrm{M}$ of CGRP significantly induced the migration of MSCs (Fig. 6B). HGF at $10 \mathrm{ng} / \mathrm{mL}$ also significantly induced the migration of MSCs. The combination of $10^{-8}-\mathrm{M}$ CGRP and $10-\mathrm{ng} / \mathrm{mL} \mathrm{HGF}$ further promoted the migration of MSCs (Fig. 7AB).

\section{Discussion}

We could detect MSCs in synovial fluid (Post). Cells derived from synovial fluid formed cell colonies consisting of spindle-shaped cells and were positive for CD44, CD73, CD90, and CD105, negative for CD31 and CD45, and had multipotentiality. These properties were identical to those of MSCs.(23)

Assuming the colony number is the MSC number, MSCs in synovial fluid (Pre) were detected in only 1 among 7 donors. A previous study reported that the mean MSC number per synovial fluid was $253(/ \mathrm{mL})$ in 22 donors with traumatic meniscus injury.(4) In this study, the mean MSC number per millilitre of synovial fluid (Pre) was $1(/ \mathrm{mL})$ in 7 donors with degenerative meniscus injury. In a comparison, the MSC number in the synovial fluid was much lower for degenerative meniscus injury than for traumatic meniscus injury. This may be due to differences in pathology. For example, according to Brophy, 
compared to a torn degenerative meniscus, a torn traumatic meniscus expressed higher in MMP-1 and MMP- $3,{ }^{4}$ which have important physiological roles in cell migration, invasion, and proliferation. $(17,24)$ A degenerative meniscus tear is considered more difficult to heal spontaneously compared to a traumatic meniscus tear. $(25,26)$ This difference may be due to the difference in the MSC number in the synovial fluid after meniscus injury.

The number of MSCs was significantly higher in the synovial fluid (Post) than in the synovial fluid (Pre). This indicates that arthroscopic procedures for harvest of synovium and meniscus repair increased MSCs in the synovial fluid in 2 weeks.

The mean MSC number per donor in synovial fluid (Post) was $432(=36 \times 3 \times 4)$. For a degenerative tear of the medial meniscus, we transplanted autologous synovial MSCs onto the repaired meniscus.(15) The number of MSCs for transplantation was $50 \times 10^{6}$ on average.(15) Assuming that the colony formation rate is $50 \%$,(27) $25 \times 10^{6}$ colony-forming cells were transplanted. This suggests that the number of MSCs we transplanted was $58 \times 10^{3}\left(=25 \times 10^{6} / 432\right)$-fold higher than that of MSCs in the synovial fluid (Post). The contribution of endogenous MSCs in the synovial fluid after harvest of synovium and meniscus suture will be much lower than transplantation of synovial MSCs for repaired meniscus with degenerative injury.

The signal intensity of CGRP correlated with the colony number per dish in 7 donors, and CGRP promoted migration of synovial fluid MSCs. CGRP is a neuropeptide released from $\mathrm{C}$ nerve fibers and not only has a pain-transmitting action but also is involved in neuropathic inflammation. $(28,29)$ The content of CGRP was determined according to previous studies about the concentration of CGRP in the knee joint. $(30,31)$ Regarding CGRP related to MSCs, Zhang et al. demonstrated that CGRP at $10^{-6}-10^{-9} \mathrm{M}$ promoted migration of human umbilical cord MSCs and that CGRP was a key chemokine in the migration of umbilical cord MSCs to the spinal lesion site in rats.(32) Chen et al. reported that $10^{-6}-10^{-10} \mathrm{M}$ CGRP promoted proliferation of bone marrow MSCs in a dose-dependent manner and that CGRP played a role in adjusting bone mass and strength in ovariectomized rats.(33) In the current study, HGF signal intensity was also correlated with colony number per dish in 7 donors, and HGF promoted migration of synovial fluid MSCs. HGF is secreted by mesenchymal cells and has effects for morphogenesis, angiogenesis, inflammation, tissue repair, etc. on various cells.(34) The HGF content was determined according to previous studies about the concentration of HGF in knee joints of primary OA patients.(35) Regarding HGF related to MSCs, Ponte et al. reported that 50-ng/mL HGF promoted migration of TNFa-primed bone marrow MSCs.(19) We first revealed the chemotactic effect of CGRP and HGF on synovial fluid MSCs. These 2 factors acted additively, suggesting different receptors and signaling pathways for $\operatorname{CGRP}(36)$ and HGF.(37)

We examined the relationship between the MSC number and protein concentration in synovial fluid. The 2 factors that were highly correlated were CGRP and HGF, which were confirmed to promote the migration of synovial fluid MSCs. In addition, these factors have already been reported to promote the migration of MSCs, though they were not found to derive from synovium. $(32,33)$ This suggests that other factors that 
showed a high relationship between the MSC number and protein concentration in the synovial fluid might also promote the migration of synovial fluid MSCs in the migration assay we performed.

We propose three limitations. First, when we aspirated synovial fluid, we did not do pumping, for which we would have injected $20 \mathrm{~mL}$ of saline and aspirated native synovial fluid with $20 \mathrm{~mL}$ of saline. If we had performed pumping, we might have aspirated a higher amount of synovial fluid. Second, of the 8 factors with a correlation coefficient between the colony number and expression level of 0.7 or higher, only CGRP and HGF were investigated for the migration assay. The other 6 factors may also promote the migration of synovial fluid MSCs. Third, the migration assay does not completely mimic the biological phenomenon in which MSCs are induced from the synovium into the synovial fluid. We do not know whether CGRP and HGF really mobilize MSCs from the synovium into the synovial fluid unless we inject CGRP and HGF into the knee joint and quantify MSCs in the synovial fluid.

\section{Conclusions}

In conclusion, MSCs in synovial fluid were hardly seen in knees with degenerated meniscus injury. Though they increased 2 weeks after harvest of synovium and meniscus repair, the number of MSCs in the synovial fluid was much lower than that of synovial MSCs in the case of transplantation in clinical situations. Both CGRP and HGF in synovial fluid could possibly induce MSCs from the synovium into the synovial fluid.

\section{Abbreviations}

MSCs

Mesenchymal stem cells; CGRP:calcitonin gene-related peptide; HGF:hepatocyte growth factor; OA:osteoarthritis; a-MEM:a-modified essential medium; HBSS:Hank's balanced salt solution; BMP-2:bone morphogenetic protein 2

\section{Declarations}

\section{Ethical approval and consent to participate}

This study was approved by the Medical Research Ethics Committee of Tokyo Medical and Dental University, and informed consent was obtained from all study subjects.

\section{Consent for publication}

All indivisuals consented to publication.

\section{Availability of data and materials}

Not applicable. 


\section{Competing interests}

The authors declare that they have no competing interests.

\section{Funding}

Not applicable.

\section{Authors' contributions}

All authors were involved in drafting the article or critically revising it for important intellectual content, and all authors approved the final manuscript. I.S. had full access to all study data and takes responsibility for the integrity of the data and the accuracy of the data analysis. N.W., K.E., and I.S. designed and performed the research, analyzed and interpreted the data, and drafted the manuscript. N.W. performed the experiments and collected the data. K.K. made substantial contributions to multidifferentiation analysis, flowcytometry, and protein array analysis. N.O., M.M., K.O., H.Ka., and Y.K. made substantial contributions to the analysis and interpretation of the data. K.T. made substantial contributions to the study design for migration assay. H.Ko. performed arthroscopy and collected synovial fluid from the patients.

\section{Acknowledgments}

We thank Ms. Mika Watanabe and Ms. Kimiko Takanashi for the management of our laboratory and Tina Hilling-_-_ for English editing.

\section{References}

1. Crevensten G, Walsh AJ, Ananthakrishnan D, Page P, Wahba GM, Lotz JC, et al. Intervertebral disc cell therapy for regeneration: mesenchymal stem cell implantation in rat intervertebral discs. Ann Biomed Eng. 2004;32(3):430-4.

2. Morito T, Muneta T, Hara K, Ju YJ, Mochizuki T, Makino H, et al. Synovial fluid-derived mesenchymal stem cells increase after intra-articular ligament injury in humans. Rheumatology. 2008;47(8):113743.

3. Sekiya I, Ojima M, Suzuki S, Yamaga M, Horie M, Koga H, et al. Human mesenchymal stem cells in synovial fluid increase in the knee with degenerated cartilage and osteoarthritis. J Orthop Res. 2012;30(6):943-9.

4. Matsukura Y, Muneta T, Tsuji K, Koga H, Sekiya I. Mesenchymal stem cells in synovial fluid increase after meniscus injury. Clin Orthop Relat Res. 2014;472(5):1357-64. 
5. Horie M, Sekiya I, Muneta T, Ichinose S, Matsumoto K, Saito H, et al. Intra-articular Injected synovial stem cells differentiate into meniscal cells directly and promote meniscal regeneration without mobilization to distant organs in rat massive meniscal defect. Stem Cells. 2009;27(4):878-87.

6. Hatsushika D, Muneta T, Nakamura T, Horie M, Koga H, Nakagawa $Y$, et al. Repetitive allogeneic intraarticular injections of synovial mesenchymal stem cells promote meniscus regeneration in a porcine massive meniscus defect model. Osteoarthritis Cartilage. 2014;22(7):941-50.

7. Hatsushika D, Muneta T, Horie M, Koga H, Tsuji K, Sekiya I. Intraarticular injection of synovial stem cells promotes meniscal regeneration in a rabbit massive meniscal defect model. J Orthop Res. 2013;31(9):1354-9.

8. Kondo S, Muneta T, Nakagawa Y, Koga H, Watanabe T, Tsuji K, et al. Transplantation of autologous synovial mesenchymal stem cells promotes meniscus regeneration in aged primates. J Orthop Res. 2017;35(6):1274-82.

9. Nakagawa Y, Muneta T, Kondo S, Mizuno M, Takakuda K, Ichinose S, et al. Synovial mesenchymal stem cells promote healing after meniscal repair in microminipigs. Osteoarthritis Cartilage. 2015;23(6):1007-17.

10. Bin SI, Kim JM, Shin SJ. Radial tears of the posterior horn of the medial meniscus. Arthroscopy. 2004;20(4):373-8.

11. Herrlin SV, Wange PO, Lapidus G, Hållander M, Werner S, Weidenhielm L. Is arthroscopic surgery beneficial in treating non-traumatic, degenerative medial meniscal tears? A five year follow-up. Knee Surg Sports Traumatol Arthrosc. 2013;21(2):358-64.

12. Beaufils $P$, Becker R, Kopf $S$, Englund M, Verdonk R, Ollivier M, et al. Surgical management of degenerative meniscus lesions: the 2016 ESSKA meniscus consensus. Knee Surg Sports Traumatol Arthrosc. 2017;25(2):335-46.

13. Englund M, Roemer FW, Hayashi D, Crema MD, Guermazi A. Meniscus pathology, osteoarthritis and the treatment controversy. Nat Rev Rheumatol. 2012;8(7):412-9.

14. Kopf $S$, Beaufils $P$, Hirschmann MT, Rotigliano N, Ollivier M, Pereira $\mathrm{H}$, et al. Management of traumatic meniscus tears: the 2019 ESSKA meniscus consensus. Knee Surg Sports Traumatol Arthrosc. 2020.

15. Sekiya I, Koga H, Otabe K, Nakagawa Y, Katano H, Ozeki N, et al. Additional Use of Synovial Mesenchymal Stem Cell Transplantation Following Surgical Repair of a Complex Degenerative Tear of the Medial Meniscus of the Knee: A Case Report. Cell Transplant. 2019;28(11):1445-54.

16. Baboolal TG, Khalil-Khan A, Theodorides AA, Wall O, Jones E, McGonagle D. A Novel Arthroscopic Technique for Intraoperative Mobilization of Synovial Mesenchymal Stem Cells. Am J Sports Med. 2018;46(14):3532-40.

17. Brophy RH, Sandell LJ, Rai MF. Traumatic and Degenerative Meniscus Tears Have Different Gene Expression Signatures. Am J Sports Med. 2017;45(1):114-20.

18. Sauerschnig M, Stolberg-Stolberg J, Schulze A, Salzmann GM, Perka C, Dynybil CJ. Diverse expression of selected cytokines and proteinases in synovial fluid obtained from osteoarthritic and healthy human knee joints. Eur J Med Res. 2014;19:65. 
19. Ponte AL, Marais E, Gallay N, Langonné A, Delorme B, Hérault O, et al. The in vitro migration capacity of human bone marrow mesenchymal stem cells: comparison of chemokine and growth factor chemotactic activities. Stem Cells. 2007;25(7):1737-45.

20. Baek SJ, Kang SK, Ra JC. In vitro migration capacity of human adipose tissue-derived mesenchymal stem cells reflects their expression of receptors for chemokines and growth factors. Exp Mol Med. 2011;43(10):596-603.

21. Qiu Y, Marquez-Curtis LA, Janowska-Wieczorek A. Mesenchymal stromal cells derived from umbilical cord blood migrate in response to complement C1q. Cytotherapy. 2012;14(3):285-95.

22. Kohno Y, Mizuno M, Ozeki N, Katano H, Otabe K, Koga H, et al. Comparison of mesenchymal stem cells obtained by suspended culture of synovium from patients with rheumatoid arthritis and osteoarthritis. BMC Musculoskelet Disord. 2018;19(1):78.

23. Mills PM, Wang Y, Cicuttini FM, Stoffel K, Stachowiak GW, Podsiadlo P, et al. Tibio-femoral cartilage defects 3-5 years following arthroscopic partial medial meniscectomy. Osteoarthritis Cartilage. 2008;16(12):1526-31.

24. Vu TH, Werb Z. Matrix metalloproteinases: effectors of development and normal physiology. Genes Dev. 2000;14(17):2123-33.

25. Beaufils P, Pujol N. Management of traumatic meniscal tear and degenerative meniscal lesions. Save the meniscus. Orthop Traumatol Surg Res. 2017;103(8S):237-S44.

26. Englund M, Guermazi A, Roemer FW, Aliabadi P, Yang M, Lewis CE, et al. Meniscal tear in knees without surgery and the development of radiographic osteoarthritis among middle-aged and elderly persons: The Multicenter Osteoarthritis Study. Arthritis Rheum. 2009;60(3):831-9.

27. Mochizuki T, Muneta T, Sakaguchi Y, Nimura A, Yokoyama A, Koga H, et al. Higher chondrogenic potential of fibrous synovium- and adipose synovium-derived cells compared with subcutaneous fatderived cells: distinguishing properties of mesenchymal stem cells in humans. Arthritis Rheum. 2006;54(3):843-53.

28. Kilo S, Harding-Rose C, Hargreaves KM, Flores CM. Peripheral CGRP release as a marker for neurogenic inflammation: a model system for the study of neuropeptide secretion in rat paw skin. Pain. 1997;73(2):201-7.

29. Onuma H, Tsuji K, Hoshino T, Inomata K, Udo M, Nakagawa Y, et al. Fibrotic changes in the infrapatellar fat pad induce new vessel formation and sensory nerve fiber endings that associate prolonged pain. J Orthop Res. 2020.

30. Larsson J, Ekblom A, Henriksson K, Lundeberg T, Theodorsson E. Immunoreactive tachykinins, calcitonin gene-related peptide and neuropeptide $Y$ in human synovial fluid from inflamed knee joints. Neurosci Lett. 1989;100(1-3):326-30.

31. Larsson J, Ekblom A, Henriksson K, Lundeberg T, Theodorsson E. Concentration of substance P, neurokinin $A$, calcitonin gene-related peptide, neuropeptide $Y$ and vasoactive intestinal polypeptide in synovial fluid from knee joints in patients suffering from rheumatoid arthritis. Scand J Rheumatol. $1991 ; 20(5): 326-35$. 
32. Zhang Y, Yang J, Zhang P, Liu T, Xu J, Fan Z, et al. Calcitonin gene-related peptide is a key factor in the homing of transplanted human MSCs to sites of spinal cord injury. Sci Rep. 2016;6:27724.

33. Chen J, Ma G, Liu W, Liu Y, Ding Y. The influence of the sensory neurotransmitter calcitonin generelated peptide on bone marrow mesenchymal stem cells from ovariectomized rats. J Bone Miner Metab. 2017;35(5):473-84.

34. Fukushima T, Uchiyama S, Tanaka H, Kataoka H. Hepatocyte Growth Factor Activator: A Proteinase Linking Tissue Injury with Repair. Int J Mol Sci. 2018;19(11).

35. Mabey T, Honsawek S, Saetan N, Poovorawan Y, Tanavalee A, Yuktanandana P. Angiogenic cytokine expression profiles in plasma and synovial fluid of primary knee osteoarthritis. Int Orthop. 2014;38(9):1885-92.

36. Cottrell GS. CGRP Receptor Signalling Pathways. Handb Exp Pharmacol. 2019;255:37-64.

37. Ilangumaran S, Villalobos-Hernandez A, Bobbala D, Ramanathan S. The hepatocyte growth factor (HGF)-MET receptor tyrosine kinase signaling pathway: Diverse roles in modulating immune cell functions. Cytokine. 2016;82:125-39.

\section{Figures}




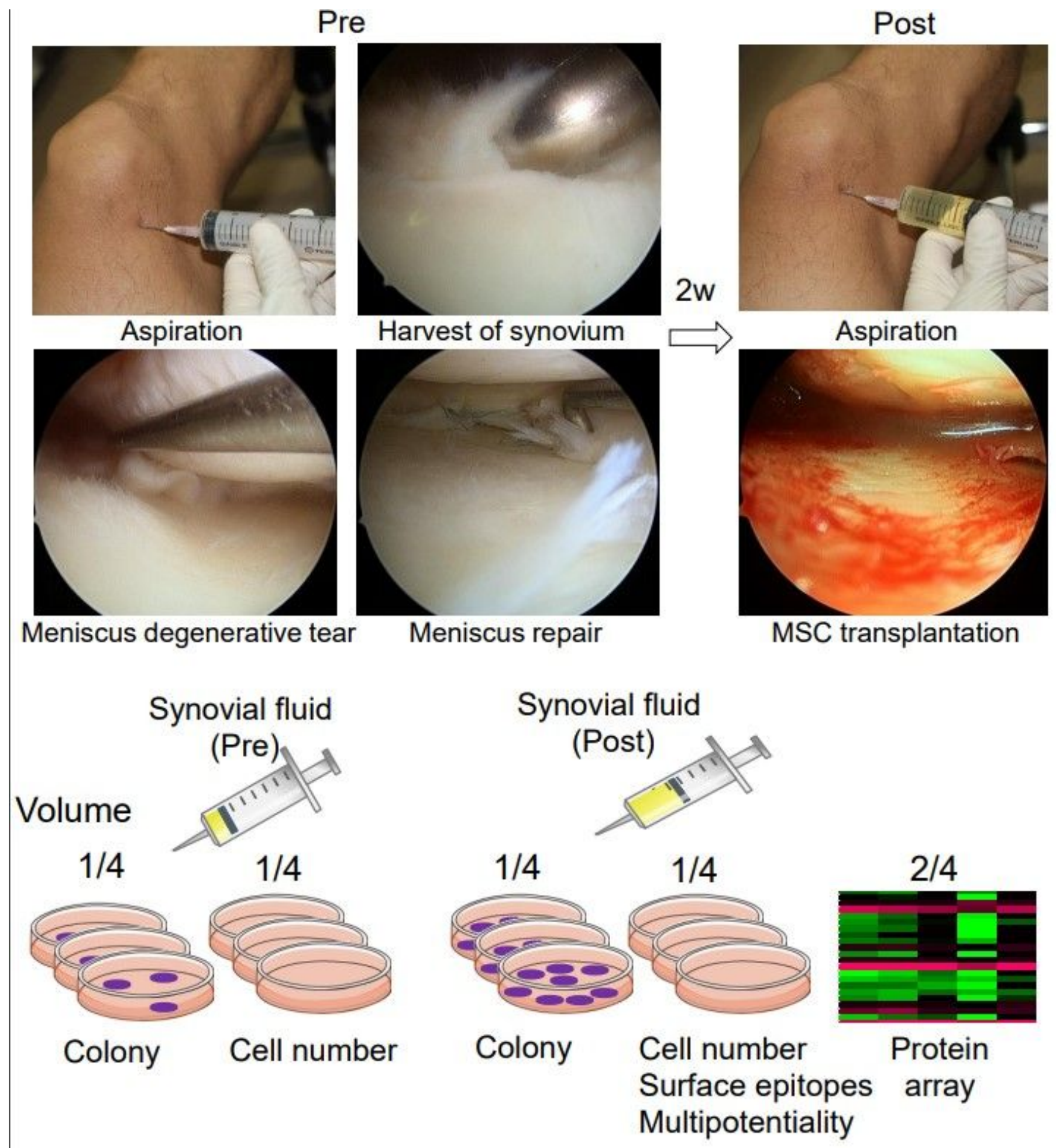

Figure 1

Schematic study design for cell cultures. Synovial fluid (Pre) was aspirated from knees with degenerative meniscus tears before harvest of synovium and meniscus repair. After 2 weeks, synovial fluid (Post) was aspirated again before transplantation of synovial MSCs. Half of the synovial fluid was plated on 6 dishes; 3 were used for colony assays, and the other 3 were used for cell number analysis, etc. The other half of synovial fluid (Post) was used for antibody array analysis. 

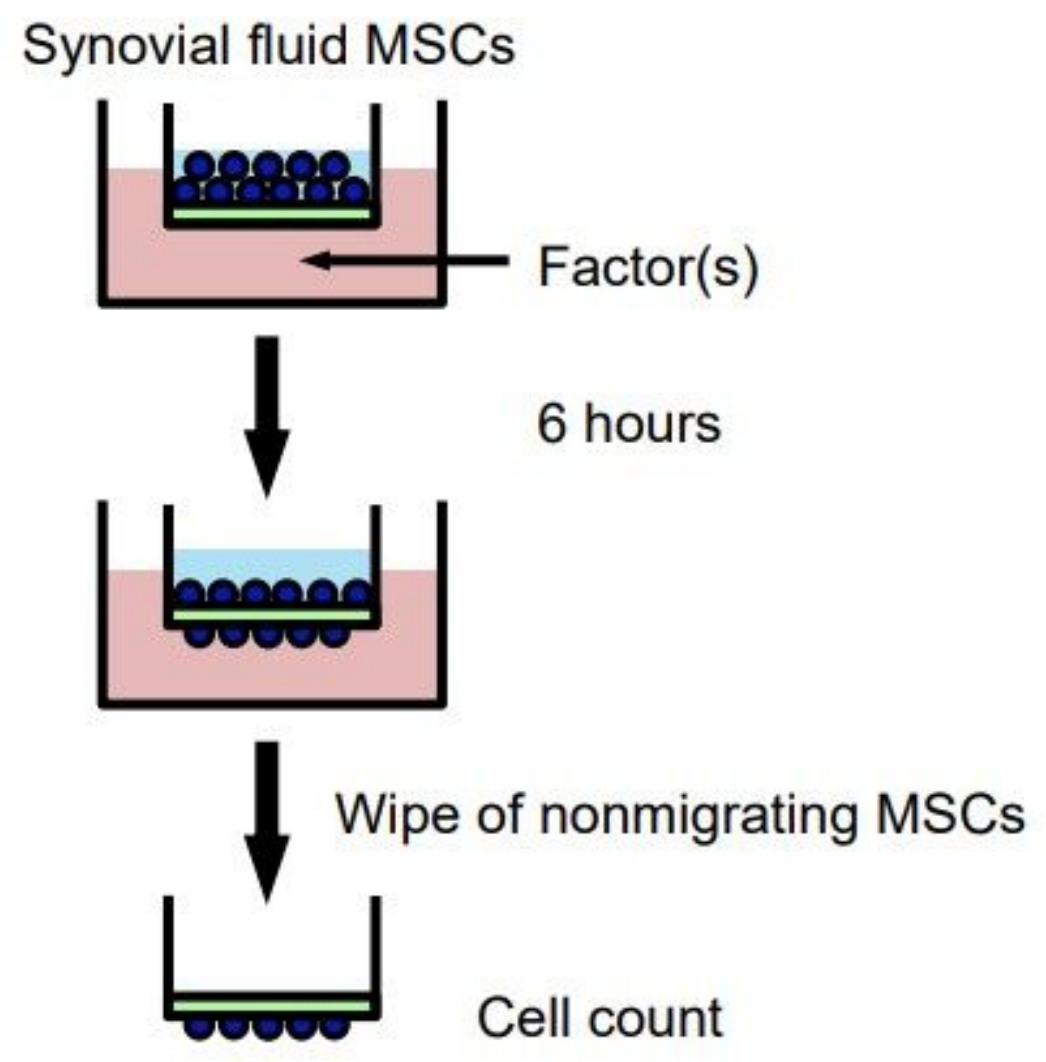

Figure 2

Schematic study design for chemotaxis assay. Synovial fluid MSCs were seeded in the upper chamber with an 8- $\mu \mathrm{m}$ Transwell microporous membrane. Factor(s) were added in the lower chamber. After 6 hours, the upper chamber was carefully removed and stained with crystal violet. MSCs that had not migrated across the pores were wiped out, and the number of MSCs on the lower side of the membrane were counted by light microscopy. 

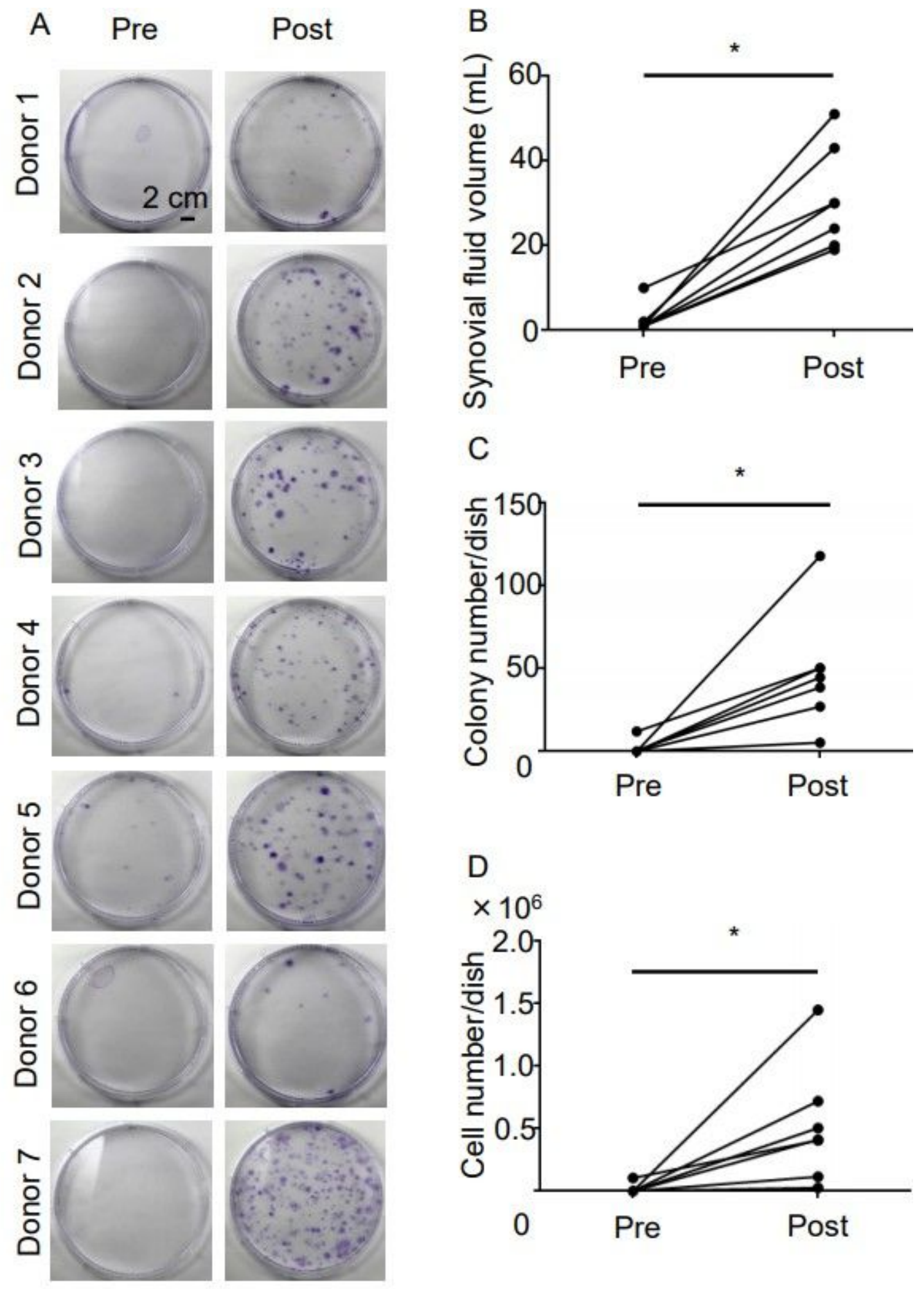

\section{Figure 3}

Analyses of synovial fluid MSCs after two weeks of culture. (A) Representative images of cell colonies stained with crystal violet. (B) Synovial fluid volume. (C) Colony number per dish. (D) Cell number per dish. ${ }^{*} p<0.05$ by the Wilcoxon matched-pairs signed-rank test $(n=7)$. 
A $\quad$ B

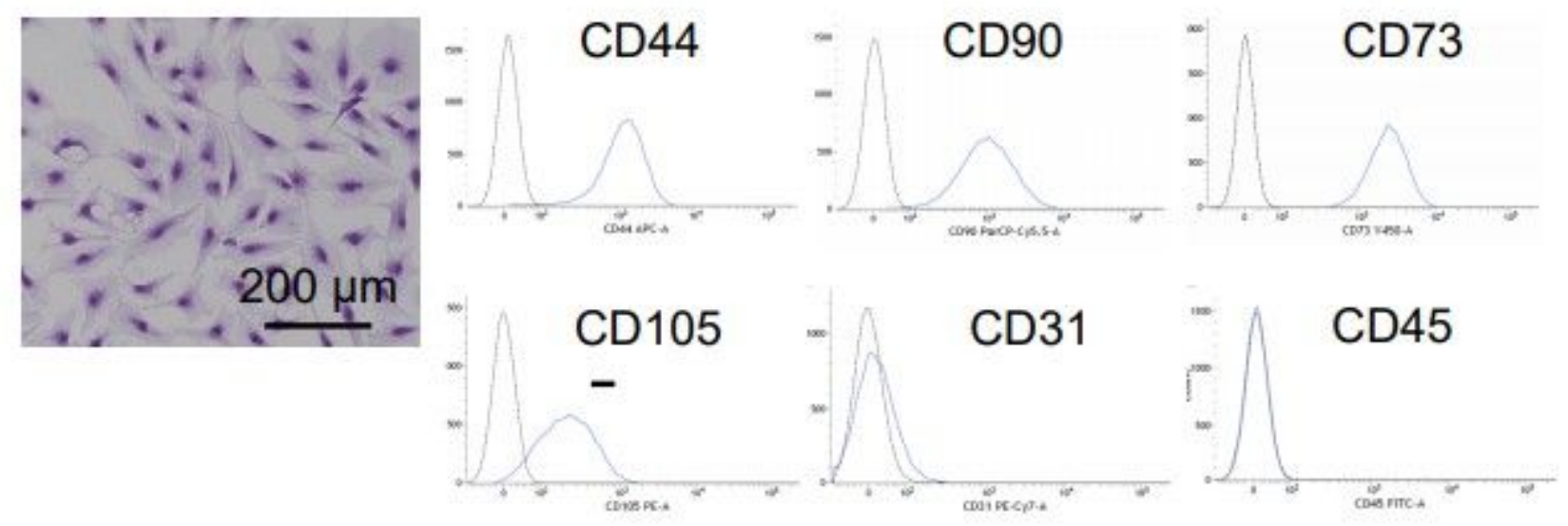

C

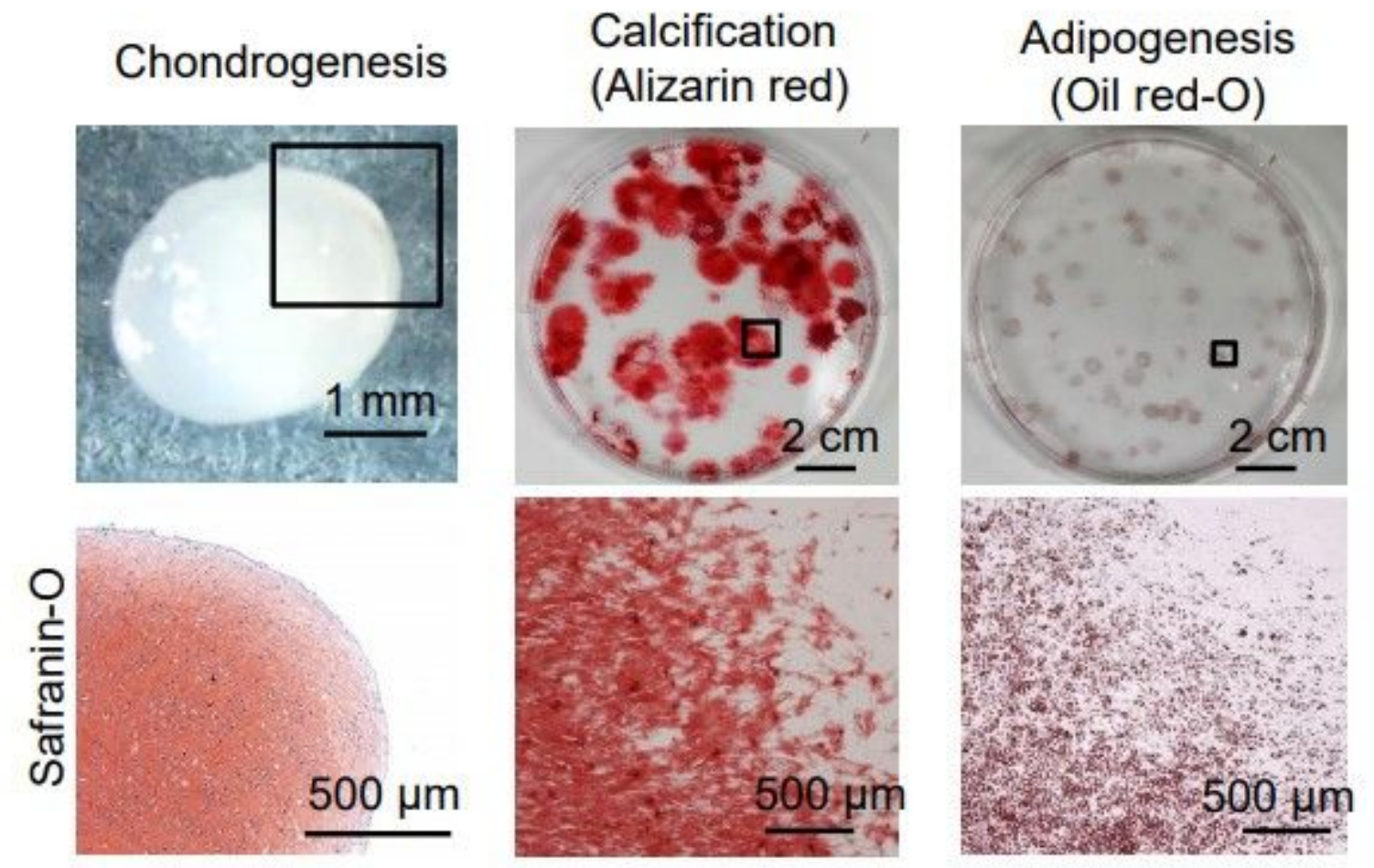

Figure 4

Properties of synovial fluid MSCs. (A) Morphology of synovial fluid MSCs stained with crystal violet. (B) Surface epitopes. (C) Multipotentiality. 


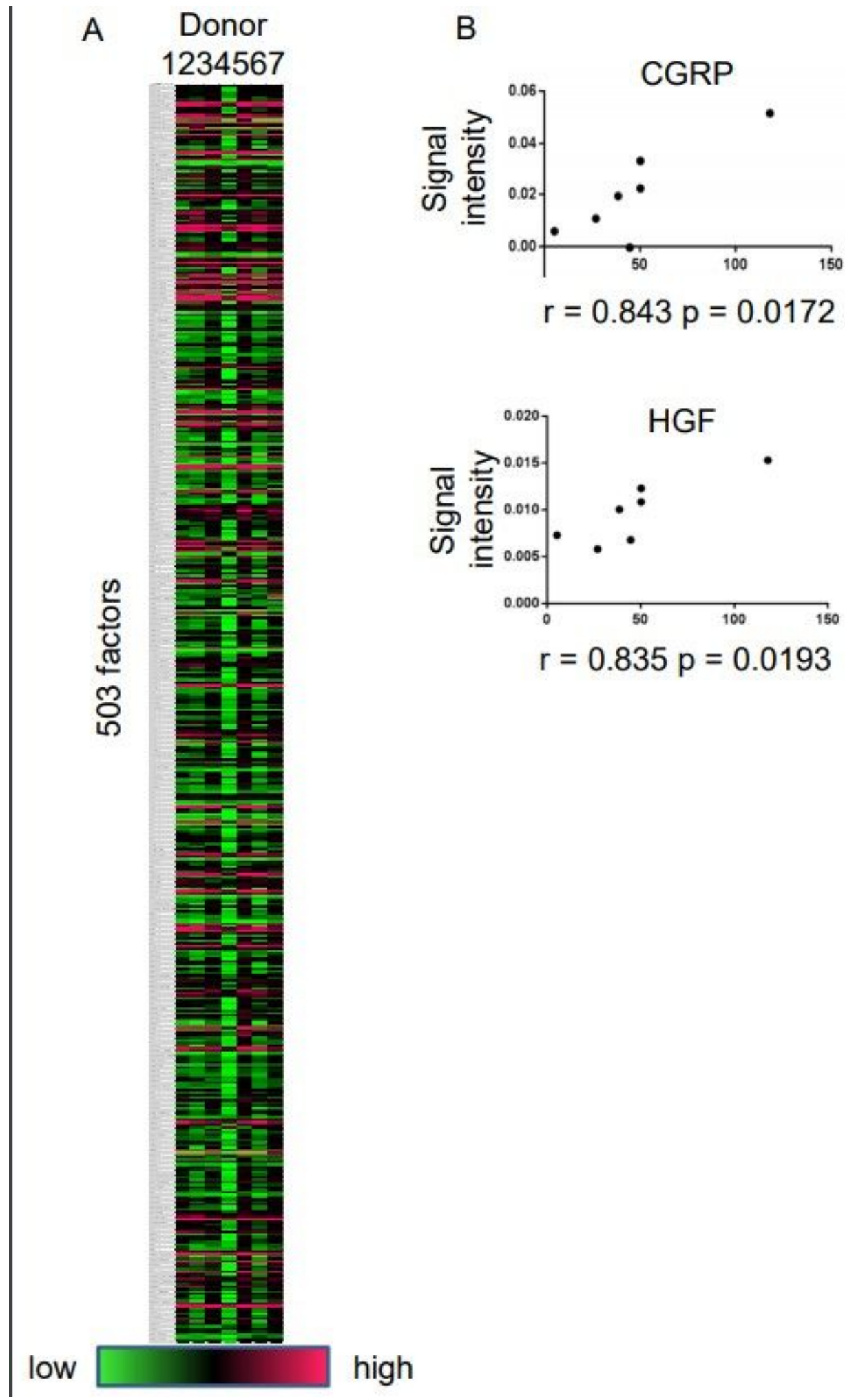

\section{Figure 5}

Relationship between expression levels in synovial fluid and colony number per dish in synovial fluid MSCs. (A) Heat map of expression levels in synovial fluid (Post) by antibody arrays in 7 donors. Green to red indicates low to high protein expression. (B) Scatter diagrams showing the relationship between colony number per dish and expression levels. Only those with a correlation coefficient exceeding 0.70 are demonstrated $(r=$ correlation coefficient, $p=$ probability, $n=7)$. 


\section{A}

$\operatorname{CGRP}(\mathrm{M})$

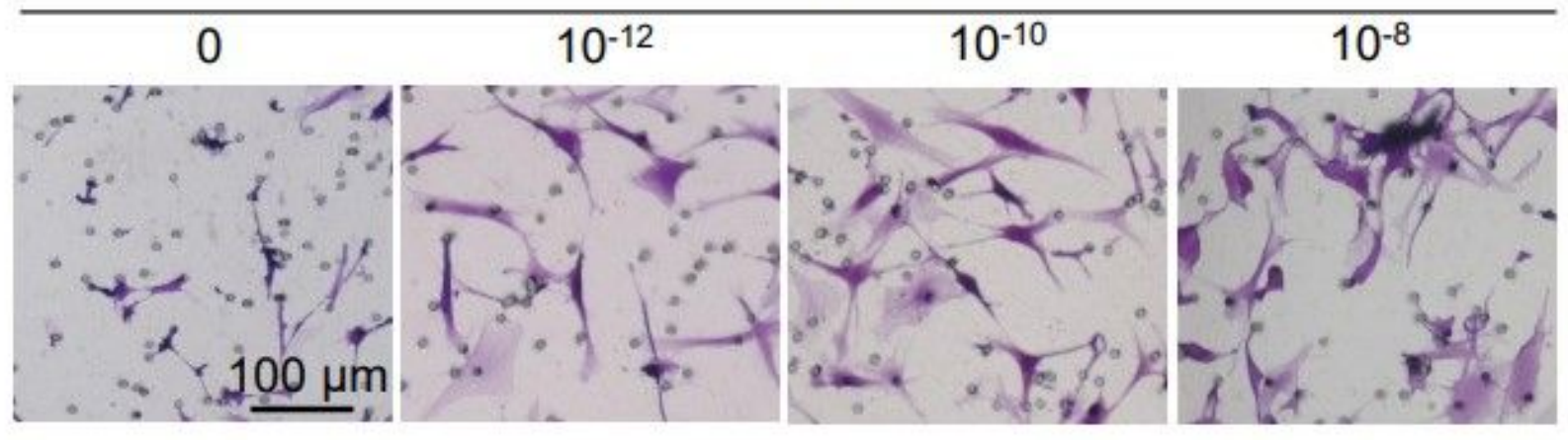

\section{HGF $(\mathrm{ng} / \mathrm{mL})$}
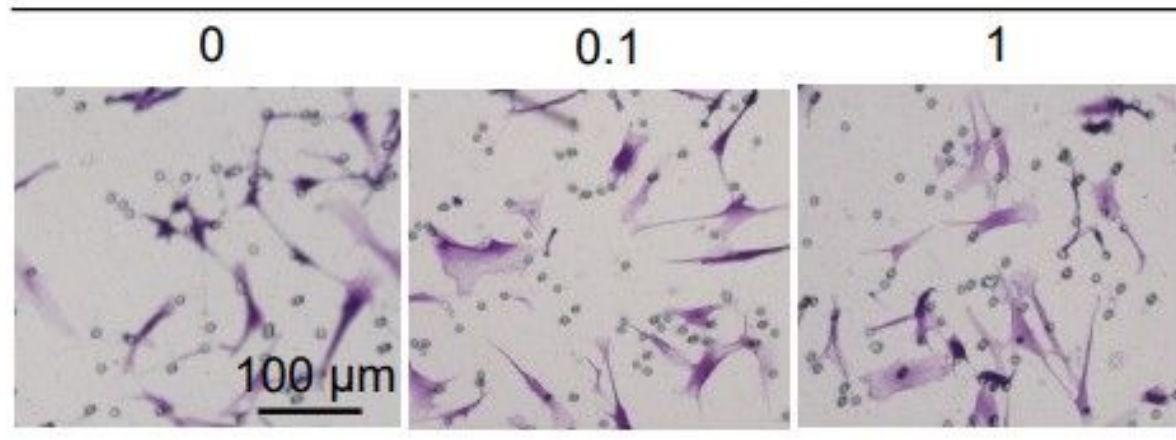

10

B
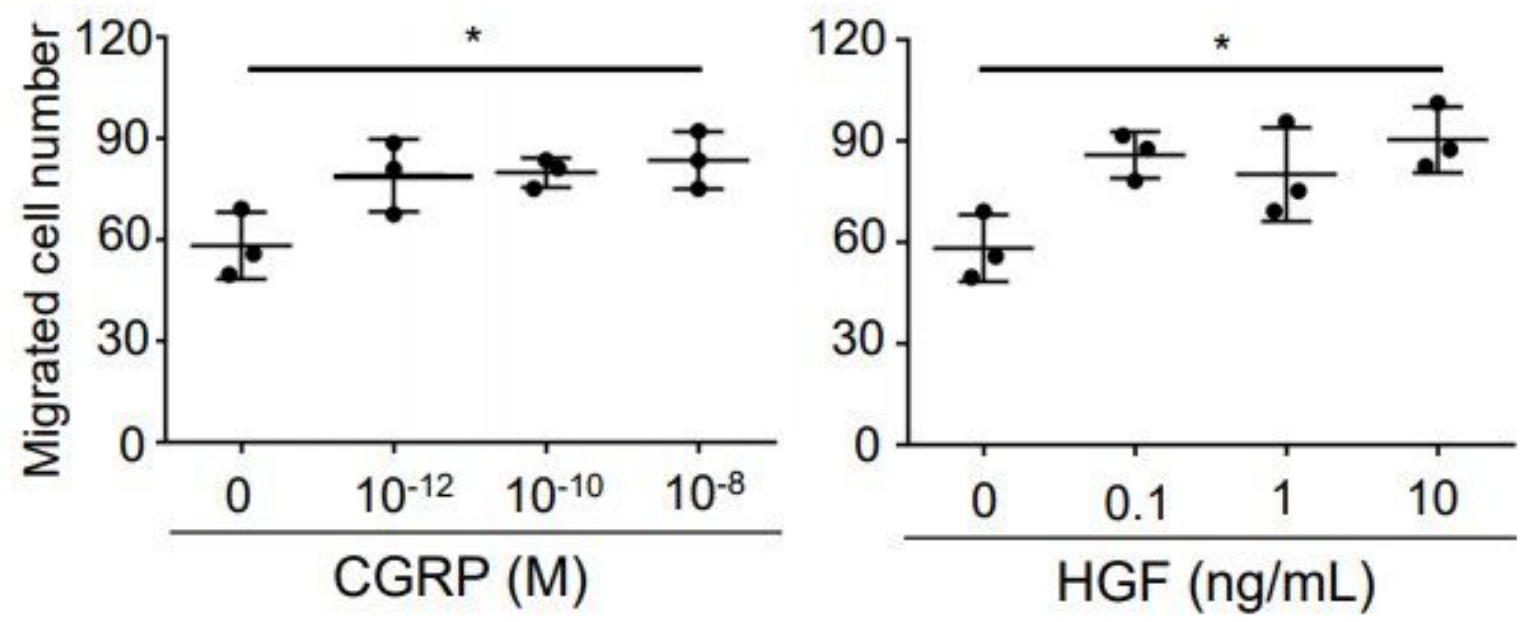

Figure 6

Chemotactic effect of CGRP alone and HGF alone on synovial fluid MSC migration. (A) Representative images of migrated cells. (B) Quantification of migrated cell number. ${ }^{*} p<0.05$ by ordinary one-way analysis of variance test followed by Holm-Sidak's multiple comparisons $(n=3)$. 
A

Control

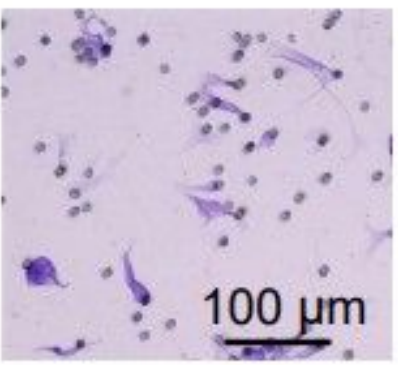

$10^{-8} \mathrm{M}$ CGRP
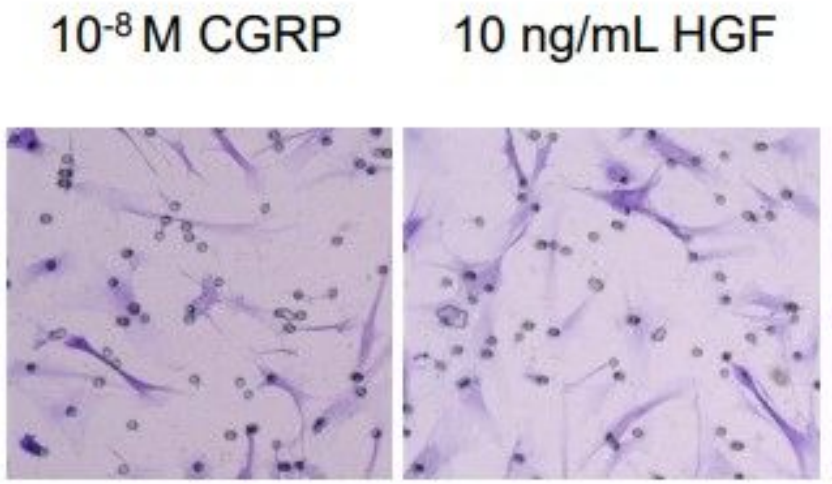

$10^{-8} \mathrm{M}$ CGRP $+$ $10 \mathrm{ng} / \mathrm{mL}$ HGF

B

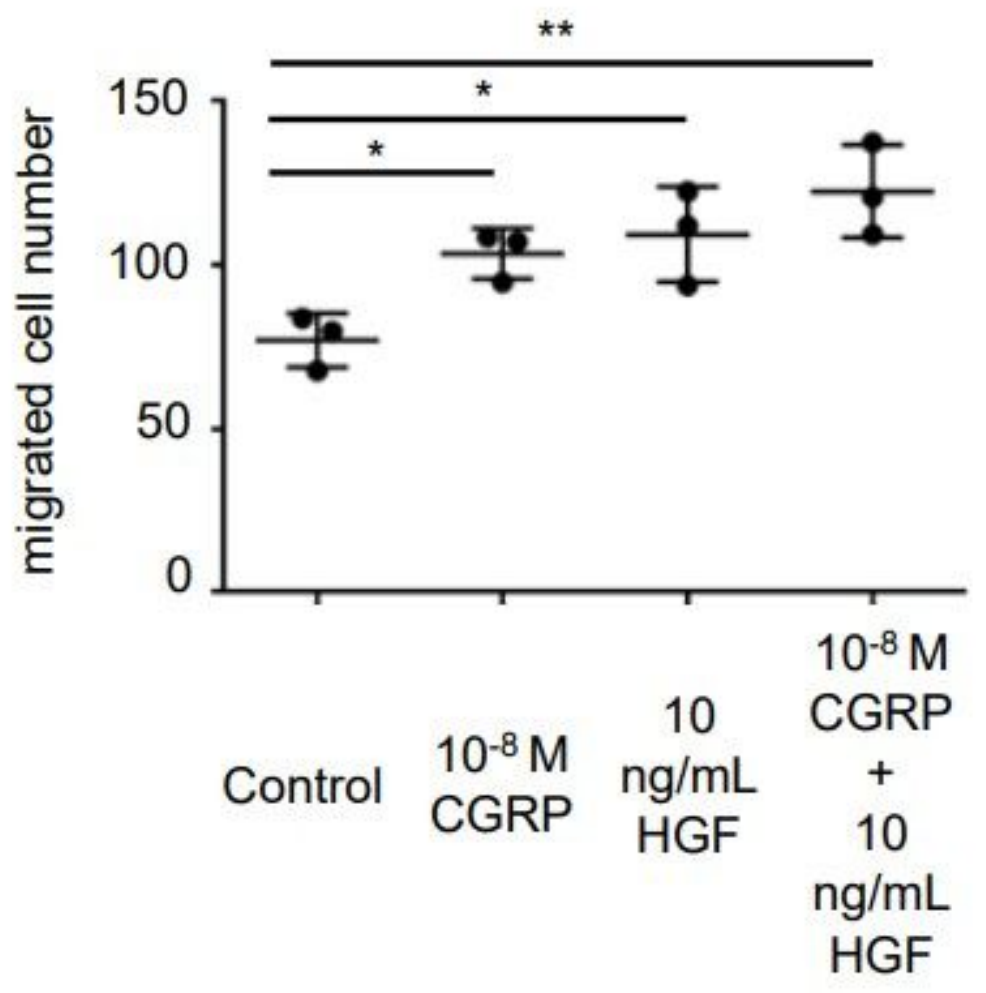

Figure 7

Chemotactic effect of the combination of CGRP and HGF on synovial fluid MSC migration. (A) Representative images of migrated cells. (B) Quantification of migrated cell number. ${ }^{*} p<0.05 ;{ }^{* *} p<0.01$ by ordinary one-way analysis of variance test followed by Holm-Sidak's multiple comparisons $(n=3)$. 